\title{
On the existence of arcs in rational curves
}

by

\author{
J. Grispolakis and E. D. Tymchatyn * (Saskatoon, Sask.)
}

Abstract. It is an open question whether curves of finite rim-type contain arcs. A. Lelek and L. Mohler gave a positive answer for the case in which the curve is hereditarily unicoherent. In this paper the question is answered in the affirmative.

1. Preliminaries. Rational curves which contain no arcs have interested topologists for several years. First, Z. Janiszewski in 1912 (see [4]) constructed an arc-like rational curve of rim-type $\omega$ which contains no arcs. A. Lelek in [6] Problem 729 asked if it is true that every curve which contains no arcs has infinite rim-type. A. Lelek and L. Mohler in [7] Theorem 1.2 proved that if $X$ is a hereditarily unicoherent curve which contains no arcs, then $X$ has infinite rim-type. B. B. Epps, Jr. in [2] constructed an arc-like curve of rim-type $n>1$ all of whose subcurves are of rimtype either 1 or $n$. J. Grispolakis and E. D. Tymchatyn gave another example in [3] of an arc-like curve of rim-type 3 all of whose subcurves are of rim-type either 1 or 3 .

In this note we answer Lelek's question in the affirmative by proving that every curve of finite rim-type contains arcs. Thus, Lelek's question is settled completely.

2. Rim-type of rational curves. A continuum is a connected, compact, metric space. A curve is a 1-dimensional continuum. If $A$ is a subset of a topological space $X$, let $A^{\prime}$ denote the derived set of $A$. Let $A^{(0)}=A$ and by transfinite induction define $A^{(\alpha)}$ for each ordinal $\alpha$, by $A^{(\alpha+1)}=\left(A^{(\alpha)}\right)^{\prime}$ and $A^{(\lambda)}=\bigcap\left\{A^{(\alpha)} \mid \alpha<\lambda\right\}$ for a limit ordinal $\lambda$. Let $\mathrm{Cl} A$ and $\mathrm{Bd} A$ denote the closure and the boundary, respectively, in $X$ of a subset $A$ of $X$. By $\operatorname{Int} A$ we denote the interior of $A$ in $X$. Let $N$ denote the set of natural numbers. If $C$ is a compact, countable subset of a metric space, then there exists a countable ordinal $\alpha$ such that $C^{(\alpha)}=\varnothing$. We call the smallest such ordinal $\alpha$ the topological type of $C$. A curve $X$ is said to be rational if $X$ admits a basis of open sets with countable boundaries. Define the rim-type of $X$ to be the smallest ordinal $\alpha$ such that $X$ has a neighbourhood basis of open sets $\left(U_{i}\right)_{i \in N}$ such that the topological type of $\mathrm{Bd} U_{i}$ is $\leqslant \alpha$ for each $i \in N$. It is well-known (see [5], p. 290) that the rim-type of a rational continuum is an ordinal number strictly smaller

* This research was supported in part by National Research Council of Canada grant No. A5616. 
than the first uncountable ordinal $\Omega$. By a mapping we shall always mean a continuous function.

The following theorem is a well-known result (see [5], p. 216) and it is stated here in the form which we shall use.

2.1. THEOREM. If $X$ is an irreducible, hereditarily decomposable continuum, then there exists a finest monotone mapping of $X$ onto the unit interval $[0,1]$. The point-inverses under this mapping are nowhere dense subcontinua of $X$ and are called the tranches of $X$.

2.2. THEOREM. Every curve of finite rim-type contains an arc.

Proof. The proof is by induction on the rim-type of the curve $X$. If rim-type of $X$ is 1 , then $X$ has a basis of open sets with finite boundaries. It is well-known that in this case $X$ is locally connected, and hence, $X$ contains an arc. Suppose that each curve of rim-type $\leqslant n-1$ contains an arc.

Just suppose that there exists a curve $X$ of rim-type $n$ such that $X$ contains no arc. Then every non-degenerate subcontinuum of $X$ has rim-type $n$. We may suppose, without loss of generality, that $X$ is an irreducible continuum. Let $\pi: X \rightarrow[0,1]$ be a finest monotone mapping of $X$ onto $[0,1]$. We may suppose since $X$ is not an arc that $\pi^{-1}(1)$ is non-degenerate. Let $T_{0}=\pi^{-1}(1)$ and let $Y_{0}=X$.

Let $\mathscr{U}$ be a countable basis of open sets for $X$ whose boundaries are pairwise disjoint and have topological type $\leqslant n$. This is possible since $X$ is compact, $\mathscr{U}$ is countable, and the boundaries of the members of $\mathscr{U}$ are zero-dimensional. We may also suppose that if $U \in \mathscr{U}$ and $x \in \mathrm{Bd} U$, then $x \in \mathrm{Cl}(X \backslash \mathrm{Cl} U)$. Let $\left\{U_{1}, U_{2}, \ldots\right\}$ be the members of $\mathscr{U}$ which meet $T_{0}$.

For each $m=1,2, \ldots$ let $D_{m}=\left(T_{0} \cap \operatorname{Bd} U_{m}\right) \backslash\left(\operatorname{Bd} U_{m}\right)^{\prime}$. Then $D_{m}$ is a discrete set for each $m=1,2, \ldots$ Let $D_{m}=\left\{x_{m 1}, x_{m 2}, \ldots\right\}$ for each $m=1,2, \ldots$ Let $Y_{1}$ be the compactification of $Y_{0} \backslash D_{1}$ which is larger than $Y_{0}$ and such that if $g_{1}: Y_{1} \rightarrow Y_{0}$ is the extension over $Y_{1}$ of the inclusion of $Y_{0} \backslash D_{1} \subset Y_{1}$ into $Y_{0}$, then $g_{1}^{-1}\left(x_{1 i}\right)$ $=\left\{y_{1 i}, z_{1 i}\right\}$. A basic open neighbourhood of $y_{1 i}$ (respectively, $\left.z_{1 i}\right)$ is given by $g_{1}^{-1}\left(U \cap U_{1}\right) \cup\left\{y_{1 i}\right\}$ (respectively, $\left.g_{1}^{-1}\left(U \backslash \mathrm{Cl} U_{1}\right) \cup\left\{z_{1 i}\right\}\right)$, where $U$ is a neighbourhood of $x_{1 i}$ in $Y_{0}$. Then $Y_{1}$ is an irreducible continuum of rim-type $n$ and $g^{-1}\left(T_{0}\right)=T_{1}$ is a tranche of $Y_{1}$. Notice that the topological type of $T_{1} \cap \mathrm{Bd}\left[\mathrm{Cl}\left(g_{1}^{-1}\left(U_{1}\right)\right)\right]$ is less than or equal to $n-1$ since $g_{1}$ maps $T_{1} \cap \mathrm{Bd}\left[\mathrm{Cl}\left(g_{1}^{-1}\left(U_{1}\right)\right)\right]$ homeomorphically onto a subset of $\left(\mathrm{Bd} U_{1}\right)^{\prime}$. Also $g_{1}$ maps $\operatorname{Bd}\left[\mathrm{Cl}\left(g_{1}^{-1}\left(U_{1}\right)\right)\right]$ homeomorphically onto $\mathrm{Bd} U_{1} \backslash D_{1}$. We identify points and subsets of $Y_{0} \backslash D_{1}$ with their preimages in $Y_{1}$.

Suppose that $m$ is a positive integer and $Y_{1}, \ldots, Y_{m-1}$ are irreducible continua of rim-type $n, g_{i}: Y_{i} \rightarrow Y_{i-1}$ is a mapping of $Y_{i}$ onto $Y_{i-1}, g_{i}$ maps $Y_{i} \backslash g_{i}^{-1} \circ \ldots \circ g_{1}^{-1}\left(D_{i}\right)$ homeomorphically onto $Y_{i-1} \backslash g_{i-1}^{-1} \circ \ldots \circ g_{1}^{-1}\left(D_{i}\right), g_{i}$ is two-to-one at the points of $g_{i-1}^{-1} \circ \ldots \circ g_{1}^{-1}\left(D_{i}\right)$ and the topological type of $T_{i} \cap \mathrm{Bd}\left[\mathrm{Cl}\left(g_{i}^{-1} \circ \ldots \circ g_{1}^{-1}\left(U_{i}\right)\right)\right]$ is at most $n-1$, where $T_{i}=g_{i}^{-1}\left(T_{i-1}\right)$ for each $i=1, \ldots, m-1$. We identify points and subsets in $Y_{i-1} \backslash g_{i-1}^{-1} \circ \ldots \circ g_{1}^{-1}\left(D_{i}\right)$ with their preimages under $g_{i}$ for each $i=1, \ldots, m-1$.
Let $Y_{m}$ be the compactification of $Y_{m-1} \backslash D_{m}$ that is larger than $Y_{m-1}$ and such that if $g_{m}: Y_{m} \rightarrow Y_{m-1}$ is the extension over $Y_{m}$ of the inclusion of $Y_{m-1} \backslash D_{m} \subset Y_{m}$ into $Y_{m-1}$, then $g_{m}^{-1}\left(x_{m i}\right)=\left\{y_{m i}, z_{m i}\right\}$. A basic open neighbourhood of $y_{m i}$
(respectively, $\left.z_{m i}\right)$ is given by

$$
g_{m}^{-1}\left(U \cap g_{m-1}^{-1} \circ \ldots \circ g_{1}^{-1}\left(U_{m}\right)\right) \cup\left\{y_{m i}\right\}
$$

(respectively, $\left.g_{m}^{-1}\left[U \backslash \mathrm{Cl}\left(g_{m-1}^{-1} \circ \ldots \circ g_{1}^{-1}\left(U_{m}\right)\right)\right] \cup\left\{z_{m i}\right\}\right)$, where $U$ is a neighbourhood of $x_{m i}$ in $Y_{m-1}$. We identify points and subsets of $Y_{m-1} \backslash D_{m}$ with their preimages in $Y_{m}$ under $g_{m}$. Then $Y_{m}$ is an irreducible continuum of rim-type $n$ and $g_{m}^{-1}\left(T_{m-1}\right)=T_{m}$ is a tranche of $Y_{m}$. Notice that the topological type of $T_{m} \cap \mathrm{Bd}\left[\mathrm{Cl}\left(g_{m}^{-1} \circ \ldots \circ g_{1}^{-1}\left(U_{m}\right)\right)\right]$ is less than or equal to $n-1$ since it is mapped by $g_{m} \circ \ldots \circ g_{1}$ homeomorphically onto a subset of $\left(\operatorname{Bd} U_{m}\right)^{\prime}$. Also $g_{1} \circ \ldots \circ g_{m}$ maps $\operatorname{Bd}\left[\mathrm{Cl}\left(g_{m}^{-1}, \ldots \circ g_{1}^{-1}\left(U_{m}\right)\right)\right]$ homeomorphically onto $\operatorname{Bd} U_{m} \backslash D_{m}$. By induction, $Y_{m}$ and $g_{m}$ are defined for each $m=1,2, \ldots$

Consider the inverse system $\left\{Y_{m}, g_{m}, N\right\}$ and let $Y_{\infty}=\left\{Y_{m}, g_{m}, N\right\}$ and let $g: Y_{\infty} \rightarrow Y_{0}=X$ be the mapping induced by the inverse limit. Then $g$ is oneto-one except at the points $x_{m i}$, for each $m, i=1,2, \ldots$, where $g$ is two-to-one, and $g$ maps $g^{-1} \circ \pi^{-1}([0,1))$ homeomorphically onto $\pi^{-1}([0,1))$. We also have that $g^{-1} \circ \pi^{-1}([0,1))$ is dense in $Y_{\infty}, Y_{\infty}$ is irreducible, and $T_{\infty}=\underset{L}{L}\left\{T_{m}, g_{m} \mid T_{m}, N\right\}$ is a tranche of $Y_{\infty}$.

If $x \in Y_{\infty} \backslash T_{\infty}$, then a basic neighbourhood of $x$ in $Y_{\infty}$ (see [1], p. 218) is given by $g^{-1}(U)$ for some $U \in \mathscr{U}$ such that $\mathrm{Cl} U \cap T_{0}=\varnothing$. Then $\operatorname{Bd}\left(g^{-1}(U)\right)$ is homeomorphic to $\mathrm{Bd} U$. If $x \in T_{\infty}$ but $x \neq x_{m i}$ for each $m, i=1,2, \ldots$, then a basic neighbourhood of $x$ in $Y_{\infty}$ is of the form $\operatorname{Int}\left[\mathrm{Cl}\left(g^{-1}\left(U_{i}\right)\right)\right]$ for some $U_{i} \in \mathscr{U}$. Then

$$
\mathrm{Bd}\left[\operatorname{Int}\left(\mathrm{Cl}\left(g^{-1}\left(U_{i}\right)\right)\right)\right]=\mathrm{Bd}\left[\mathrm{Cl}\left(g^{-1}\left(U_{i}\right)\right)\right]
$$

is homeomorphic to $\mathrm{Bd}\left[\mathrm{Cl}\left(g_{i}^{-1} \circ \ldots \circ g_{1}^{-1}\left(U_{i}\right)\right)\right]$ and the latter set has topological type less than or equal to $n$. Also, $T_{\infty} \cap \mathrm{Bd}\left[\mathrm{Cl}\left(g^{-1}\left(U_{i}\right)\right)\right]$ is homeomorphic to $\operatorname{Bd}\left[\mathrm{Cl}\left(g_{i}^{-1} \circ \ldots \circ g_{1}^{-1}\left(U_{i}\right)\right)\right] \cap T_{\infty}$. If $x=y_{m i}$ (respectively, $\left.x=z_{m i}\right)$, then a basic neighbourhood $G$ of $x$ is of the form $\operatorname{Int}\left[\mathrm{Cl}\left(g^{-1}\left(U_{m} \cap U_{j}\right)\right)\right] \cup\left\{y_{m i}\right\}$ (respectively, Int $\left.\left[\mathrm{Cl}\left(g^{-1}\left(U_{j} \backslash \mathrm{Cl} U_{m}\right)\right)\right] \cup\left\{z_{m i}\right\}\right)$, where $U_{j}$ is a basic neighbourhood of $g(x)$ in $Y_{0}$. The boundary of this neighbourhood in $Y_{\infty}$ is contained in

$$
\operatorname{Bd}\left(g^{-1}\left(U_{m} \cap U_{j}\right)\right) \subset \operatorname{Bd}\left(g^{-1}\left(U_{m}\right)\right) \cup \operatorname{Bd}\left(g^{-1}\left(U_{j}\right)\right)
$$

(respectively, $\mathrm{Bd}\left(g^{-1}\left(U_{j} \backslash \mathrm{Cl} U_{m}\right)\right) \subset \operatorname{Bd}\left(g^{-1}\left(U_{j}\right)\right) \cup \operatorname{Bd}\left(g^{-1}\left(U_{m}\right)\right)$ ). This shows that the boundary of the neighbourhood $G$ has topological type $\leqslant n$. Also $T_{\infty} \cap \mathrm{Bd} G$ is mapped by $g$ homeomorphically onto a subset of $\left(\operatorname{Bd} U_{m}\right)^{\prime} \cup\left(\operatorname{Bd} U_{j}\right)^{\prime}$. Hence, the rim-type of $Y_{\infty}$ is less than or equal to $n$ and the rim-type of $T_{\infty}$ is less than or equal to $n-1$. Therefore, $T_{\infty}$ contains an arc. Since $g$ is at most two-to-one $T_{0}=g\left(T_{\infty}\right)$ also contains an arc. This contradicts the assumption that $X$ does not contain any arc. The proof of the theorem is complete. 
[1] S. Eilen berg and N. Steenrod, Foundations of Algebraic Topology, Princeton University Press, Princeton 1952.

[2] B. B. Epps, Jr., Some curves of prescribed rim-types, Colloq. Math. 27 (1973), pp. 69-71.

[3] J. Grispolakis and E. D. Tymchatyn, Confluent images of rational contitua, to appear in Houston J. Math.

[4] Z. Janiszewski, Über die Begriffe "Linie" und "Fläche", Proc. Cambridge Internat. Congr. Math. 2 (1912), pp. 126-128.

[5] K. Kuratowski, Topology, Vol. II, New York-London-Warszawa 1968.

[6] A. Lelek, Some problems concerning curves, Colloq. Math. 23 (1971), pp. 93-98.

[7] - and L. Mohler, On the topology of curves III, Fund. Math. 71 (1971), pp. 147-160.

\section{DEPARTMENT OF MATHEMATICS}

UNIVERSITY OF CRETE

Iraklion, Crete

DEPARTMENT OF MATHEMATICS

UNIVERSITY OF SASKATCHEWAN

Saskatoon, Saskatchewan

Canada

\section{On compact spaces which are locally Cantor bundles}

by

\author{
Andrzej Gutek (Katowice)
}

Abstract. The paper deals with what we call the local bundles over $X$, i.e. with compact Hausdorff spaces such that each point has a neighbourhood homeomorphic to the product $X \times J$, iven totally disconnected compact Hausdorf space and $J$ is an ope the bundle $X \times[0,1]$ by identifying points $\langle x, i\rangle$ with $h\langle x, i\rangle$, where $h$ is a continuous involution without fixed points on some copies of $X \times\{0,1\}$.

1. Preliminaries. If $X$ is Hausdorf, $I$ is the unit interval $\{t \in R: 0 \leqslant t \leqslant 1\}$, and $h$ is a continuous involution on $X \times\{0,1\}$, then we denote by $X \times I / h$ the quotient of the product $X \times I$, where points $\langle x, i\rangle$ and $h\langle x, i\rangle$ are identified.

If $h$ has no fixed point, then each point of the space $X \times I / h$ has a neighbourhood homeomorphic to the product $X \times J$, where $J$ is an open interval $\{t \in R: 0<t<1\}$. If the involution $h$ is determined by a homeomorphism $f: X \rightarrow X$ in such a way that $h\langle x, 0\rangle=\langle f(x), 1\rangle$ and $h\langle x, 1\rangle=\left\langle f^{-1}(x), 0\right\rangle$, then we write $X \times I \mid f$ rather than $X \times I / h$.

LemMa. Let $X$ be a compact totally disconnected Hausdorff space, and let $D$ be a closed-open subset of $X$. Let $Y$ be a compact Hausdorff space each point of which has a neighbourhood homeomorphic to $X \times J$, where $J$ is an open unit interval. Let $Z$ be a closed 'subset of $Y$ homeomorphic to $D \times I$ under a homeomorphism $f$ and such that Int $_{Y} Z=f^{-1}(D \times J)$. Then the quotient space $Y \mid D \times I$, which is obtained from $Y$ by collapsing each arc in $Z$ to a point, is homeomorphic to $Y$.

Proof. For each point $y$ of $f^{-1}(D \times\{0\})$ take a neighbourhood $V_{y}$ homeomorphic to $X \times J$ under a homeomorphism $g_{y}$. Consider $g_{y}\left(Z \cap V_{y}\right)$ and $g_{y}\left(\right.$ Int $\left._{Y} Z \cap V_{y}\right)$. Since $Y$ is compact and Hausdorff and $V_{y}$ is an open subset of $Y$, there exist a closed-open subset $D_{y}$ of $X$ and points $a_{y}<b_{y}$ of $J$ such that $g_{y}^{-1}\left(D_{y} \times\left[a_{y}, b_{y}\right]\right)$ contains $y$ in its interior and has no point in common with $f^{-1}\left(X \times\left[\frac{1}{2}, 1\right]\right)$ and the intersection of each arc of $D_{y} \times\left[a_{y}, b_{y}\right]$ with $g_{y}\left(Z \cap V_{y}\right)$ is a proper non-degenerate subinterval of that arc. Denote the set $g_{y}^{-1}\left(D_{y} \times\left(a_{y}, b_{y}\right)\right)$ 